\title{
REEPing the benefits of an animal model of hereditary spastic paraplegia
}

\author{
Ariel Y. Deutch,1,2,3,4 Peter Hedera,3,4,5 and Roger J. Colbran',4,6
}

\begin{abstract}
${ }^{1}$ Department of Psychiatry, ${ }^{2}$ Department of Pharmacology, ${ }^{3}$ Vanderbilt Brain Institute, ${ }^{4}$ Vanderbilt Kennedy Center, ${ }^{5}$ Department of Neurology, and ${ }^{6}$ Department of Molecular Physiology and Biophysics, Vanderbilt University Medical Center, Nashville, Tennessee, USA.
\end{abstract}

\begin{abstract}
The hereditary spastic paraplegias (HSPs) are characterized by spasticity of the leg muscles due to axonal degeneration of corticospinal neurons. Beetz et al. report that the core motor phenotype and axonal pathology of HSPs are recapitulated in mice lacking the HSP-associated gene Reep1. REEP1 is shown to regulate ER structure in motor cortex neurons. The Reep1 knockout mouse should be a very useful model in which to study the mechanisms of progressive axon loss in HSPs and other disorders.
\end{abstract}

\section{Hereditary spastic paraplegias: a diverse group of disorders}

The hereditary spastic paraplegias (HSPs) are a heterogeneous group of upper motor neuron disorders marked by progressive spasticity, weakness, and hyperreflexia of the legs. In contrast to these so-called "pure" forms of HSP, "complicated" forms are marked by additional symptoms, ranging from retinal depigmentation to seizures. Autosomal dominant inheritance occurs in at least $70 \%$ of HSPs and is usually associated with the pure forms. HSP pathology is notable for pronounced degeneration of descending corticospinal neuronal axons and ascending axons in the dorsal columns (1). A limited number of postmortem studies suggest that there is little or no involvement of noncorticospinal axons in pure HSPs $(1,2)$.

Mutations in several genes have been linked to autosomal dominant HSPs, with mutations in spastin (SPG4) and atlastin (SPG3A) accounting for well over half of all cases (3-5). Mutations in the receptor expression-enhancing protein 1 (REEP1) gene have been found in a small percentage of pure HSPs (SPG31) (6-10). The mechanisms whereby REEP1 mutations lead to HSP are poorly understood.

\section{A new model of HSP}

In this issue, Beetz and colleagues (11) report the identification of a deletion of exon 2 of REEP1 isolated from a patient with familial early-onset pure HSP. Several previously identified HSP-associated

Conflict of interest: The authors have declared that no conflict of interest exists.

Citation for this article: J Clin Invest. 2013; 123(10):4134-4136. doi:10.1172/JCI72324.
REEP1 variants have been shown to result in C-terminal truncations of the protein (8-10). Beetz et al. generated a transgenic mouse in which exon 2 of Reep 1 was removed in order to examine the underlying cellular pathology and its mechanisms. This mutation caused a frame shift in the translation of exon 3, resulting in a premature stop codon. Immunoblot studies with an antibody recognizing a C-terminal epitope showed that full-length REEP1 protein is absent in mice homozygous for the mutant transgene. Accordingly, Beetz and associates designated the mice as carrying a Reep1 knockout allele. However, there remains a formal possibility that the mutated transcript may produce a truncated N-terminal fragment of the REEP1 protein, which could exert some dominant-negative action on the function of full-length REEP1.

Behavioral examination of Reep1-null mice that were less than 1 year of age did not reveal any obvious motor deficits. Older mice exhibited several changes in hind limb function, including abnormal abduction during locomotion, simultaneous forward movements of both hind limbs, and spastic clonus while climbing; however, a more rigorous quantitative analysis revealed the onset of motor deficits at an earlier time point: a change in the foot/base angle during ambulation of 4- to 5-month-old Reep1 knockout mice was observed.

Pathological examination of 13-monthold Reep $1^{-1-}$ mice did not reveal a decrease in the number of pyramidal cells in the motor cortex; however, ultrastructural studies of 7.5-month-old mice uncovered axonal deficits in the corticospinal tract, which is consistent with a retrograde axonopathy. A limited number of human postmortem studies of pure HSPs suggest that there is a progressive retrograde axonopathy of corticospinal neurons, although the apparent progressive nature of these degenerative changes may be due to sampling bias (1). More detailed studies of the development of corticospinal axonopathy in the Reep1 knockout mice should determine the precise time course of distal axon loss in HSPs and ascertain whether the progression follows a monotonic function.

\section{The function and localization of REEP1 in the ER}

Previous studies of REEP1 suggested that it localizes to mitochondria (10). In contrast, subcellular fractionation studies reported by Beetz et al. are consistent with an ER localization of REEP1. It is not clear whether the different conclusions reached regarding REEP1 localization reflect differences in antibody epitopes or cell types. More likely, perhaps, is the possibility that REEP1 is enriched at mitochondrial-ER interfaces (12) that are not preserved in brain lysates. Beetz and colleagues nicely revealed an ER function of REEP1 by demonstrating that the size of preformed liposomes (artificial membranes) is dramatically reduced by the addition of purified recombinant wild-type REEP1. The addition of a recombinant REEP1, in which key hydrophobic residues are replaced with polar residues, did not reduce the size of purified liposomes. The authors suggest that the wild-type REEP1 protein promotes liposomal membrane curvature to generate smaller liposomes. This interpretation is supported by ultrastructural studies demonstrating that REEP1 is bound and localized to the highly curved membranes of smaller liposomes.

Are these studies with liposomes and recombinant proteins relevant to complex cortical motor neurons, where multiple proteins that affect membrane budding and fusion and other ER functions are 


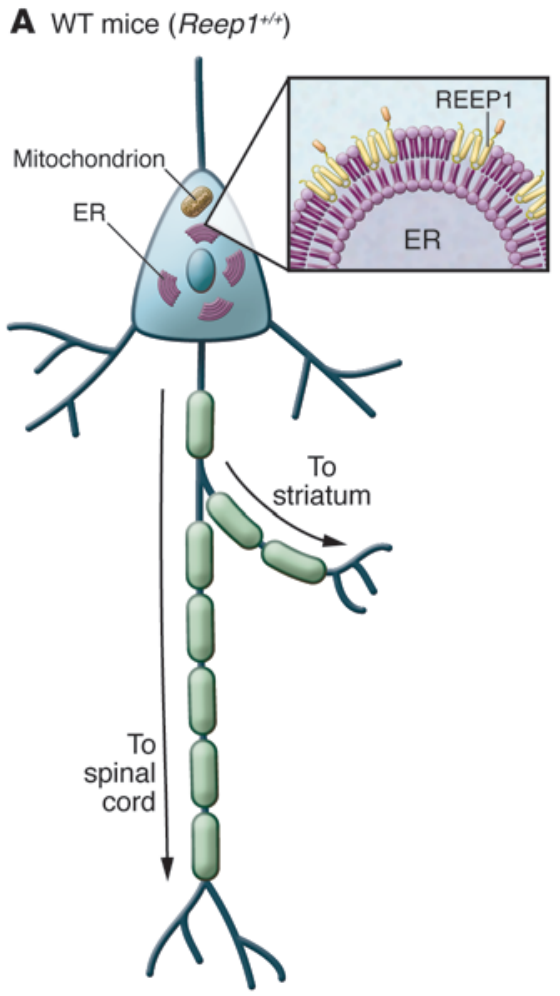

present? It seems that the answer is yes: careful EM studies of layer $\mathrm{V}$ pyramidal cells in the motor cortex of 1-year-old wildtype, Reep $1^{-/+}$, and Reep $1^{-/-}$mice revealed a REEP1 dose-dependent increase in the average length and a decrease in the number of individual ER structures. This is consistent with the hypothesis that loss of REEP1 decreases ER curvature, resulting in a reduction in the apparent number but an increase in the length of ER tubules. Serial EM reconstructions will be needed to conclusively confirm this hypothesis. The loss of REEP1 may affect ER morphology and function in multiple types of neurons, since Reep1 mRNA is highly expressed not only in the motor cortex but also in other cortices and many subcortical neurons (13). Thus, it will be informative to compare the ER morphology and function of identified populations of layer $\mathrm{V}$ motoneurons with neurons in other brain regions and even other cell types that express high levels of REEP1.

\section{Exploring common ground in HSPs}

The finding by Beetz et al. that loss of REEP1 results in a rearrangement of ER morphology suggests that REEP1 potentially affects diverse ER functions, such as $\mathrm{Ca}^{2+}$ homeostasis, vesicular trafficking, protein maturation, and ER stress responses. We began this

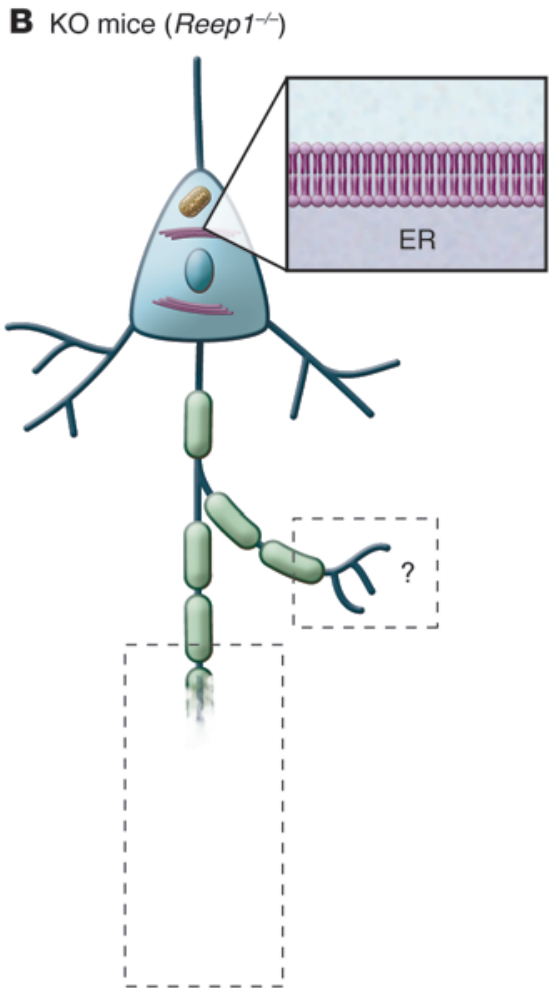

\section{Figure 1}

Schematic illustration of a corticospinal neuron in wild-type $\left(R e e p 1^{+/+}\right)$and knockout (Reep $1^{-/-}$) mice. In the knockout mouse, there is distal loss of the primary axon to the spinal cord; the status of the collateral axon to the striatum is unknown. In addition, the ER tubules in the mutant mouse are fewer in number and longer than those seen in the wild-type mouse. The inserts illustrate the effect of REEP1 on ER membrane curvature. In wild-type mice, membrane $\alpha$-helices of REEP1 (yellow) are inserted into the outer leaflet of the ER membrane, promoting curvature, with a cytosolic C-terminal microtubule-binding domain (orange). In Reep1-/mice, the lack of REEP1 protein results in more planar ER membranes. As a result, ER membranes appear much longer in electron micrographs of thin sections. The lack of ER membrane curvature is hypothesized to disrupt vesicular trafficking along microtubules in long corticospinal axons of motoneurons in Reep $1^{-1-}$ mice, resulting in progressive retrograde axonal degeneration.
Commentary by indicating that the HSPs "are a heterogeneous group of disorders." This hackneyed phrase can lead us astray by emphasizing the differences in pure HSPs rather than the common presentation of these disorders. REEP 1 joins a growing group of genes implicated in HSPs that are involved in ER morphogenesis and membrane fusion events (Figure 1), including reticulon 2, TRKfused gene, atlastin, and spastin (14-16) Additionally, another autosomal dominant pure HSP (SPG10) is attributed to a mutation in the motor protein gene KIF5A, which results in a loss of kinesin-1-mediated axonal transport of cell organelles (17). Thus, it appears that ER biology and transport may represent a final common path for the development of the pathology of HSP-associated corticospinal axon degeneration, just as the motoneuron was the final common path proposed by Sherrington (18). We await studies on the consequence of deletion of multiple HSP-associated genes to determine whether there is an additive effect on pathological and behavioral phenotypes and the rate of motor impairment.

Beetz and associates, while logically focusing on the axon, did not forget that the axon is an elaboration of the soma of corticospinal neurons. They noted subtle pathological changes in deep-layer cells of the motor cortex, although these were not definitely identified as motoneurons. It is possible that pathological changes reported to date in pure HSPs are more extensive than previously thought. There is a paucity of careful postmortem studies of the pure HSPs due in part to the indolent course of this group of disorders and the fact that even in early-onset pure HSP, there is some sparing of distal motor function. However, advances in in vivo imaging methods that allow resolution of the layers of the cortex may provide a helpful surrogate for postmortem evaluation. Similarly, more detailed behavioral assessment of individuals with pure HSPs may reveal additional neurological deficits, providing clues to the pathogenesis of HSPs. Most motor cortex neurons not only project to the cord but also give off collaterals that innervate the striatum (19). Loss of the striatal axon collateral of the motoneuron in pure HSP may lead to procedural learning deficits, which have not been examined in the HSPs. Interestingly, spastin mutations in one type of autosomal dominant HSP are associated with cognitive deficits and frontal lobe involvement (20). Animal studies as well as human studies have restricted their attention to the pyramidal tract, and it is now clear that animal models, such as the one advanced by Beetz et al. in this issue, will be useful in exploring non- 
motor phenotypes of HSPs. It also remains unclear whether pure HSPs can be linked to cell-autonomous processes or are non-cell autonomous, and indeed whether there are "protective" features in nonspinal targets of upper motoneurons that result in some sparing of neuronal function.

In summary, Beetz and colleagues have generated what promises to be a very useful mouse model of HSP. Future studies of these mice should uncover important features of the pathogenic mechanisms underlying this disease. These mice will also be useful in developing a deeper understanding of ER membrane biology. It is likely that we will "REEP" the benefit of this work for some years to come.

\section{Acknowledgments}

Supported in part by NIH grants (MH077298, to A.Y. Deutch; MH063232 and NS078291, to R.J. Colbran; and NS057666, to P. Hedera).

Address correspondence to: Ariel Y. Deutch, Vanderbilt Brain Institute, 8122 MRBIII, $46521^{\text {st }}$ Ave. South, Nashville, Tennessee 37232, USA. Phone: 615.327.7090; E-mail: ariel.deutch@vanderbilt.edu.
1. DeLuca GF, Ebers GC, Esiri MM. The extent of axonal loss in the long tracts in hereditary spastic paraplegia. Neuropath Appl Neurobiol. 2004;30(6):576-584.

2. White $\mathrm{KD}$, et al. Clinical and pathologic findings in hereditary spastic paraparesis with spastin mutation. Neurology. 2000;55(1):89-94.

3. Depienne C, Stevanin G, Brice A, Durr A. Hereditary spastic paraplegias: an update. Curr Opin Neurol. 2007;20(6):674-680.

4. Salinas S, Proukakis C, Crosby A, Warner TT. Hereditary spastic paraplegia: clinical features and pathogenetic mechanisms. Lancet Neurol. 2008;7(12):1127-1138.

5. Sauter S, et al. Mutation analysis of the spastin gene (SPG4) in patients in Germany with autosomal dominant hereditary spastic paraplegia. Hum Mutat. 2002;20(2):127-132.

6. Schlang KJ, Arning L, Epplen JT, Stemmler S. Autosomal dominant hereditary spastic paraplegia: novel mutations in the REEP1 gene (SPG31). BMC Med Genet. 2008;9:71.

7. Zuchner S, et al. Mutations in the novel mitochondrial protein REEP1 cause hereditary spastic paraplegia type 31. Am J Hum Genet. 2006; $79(2): 365-369$

8. Beetz C, et al. REEP1 mutation spectrum and genotype/phenotype correlation in hereditary spastic paraplegia type 31. Brain. 2008;131(pt 4):1078-1086.

9. Hewamadduma C, et al. New pedigrees and novel mutation expand the phenotype of REEP1-associated hereditary spastic paraplegia (HSP). Neurogenetics. 2009;10(2):105-110.

10. Goizet C, et al. REEP1 mutations in SPG31: frequency, mutational spectrum, and potential association with mitochondrial morpho-functional dysfunction. Hum Mutat. 2011;32(10):1118-1127.

11. Beetz C, et al. A spastic paraplegia mouse model reveals REEP1-dependent ER shaping. J Clin Invest. 2013;123(10):4273-4282.

12. Friedman JR, Lackner LL, West M, DiBenedetto JR, Nunnari J, Voeltz GK. ER tubules mark sites of mitochondrial division. Science. 2011;334(6054):358-362.

13. Allen Institute for Brain Science. Allen Mouse Brain Atlas. AIBS Web site. http://mouse.brainmap.org/experiment/show/74821680. Accessed August 26, 2013

14. Zhao J, Matthies DS, Botzolakis EJ, Macdonald RL, Blakely RD, Herrera P. Hereditary spastic paraplegia-associated mutations in the NIPA1 gene and its Caenorhabditis elegans homolog trigger neural degeneration in vitro and in vivo through a gain-of-function mechanism. J Neurosci. 2008; 28(51):13938-13951.

15. Park SH, Zhu PP, Parker RL, Blackstone C. Hereditary spastic paraplegia proteins REEP1, spastin, and atlastin- 1 coordinate microtubule interactions with the tubular ER network. J Clin Invest. 2010;120(4):1097-1110.

16. Montenegro G, et al. Mutations in the ER-shaping protein reticulon 2 cause the axon-degenerative disorder hereditary spastic paraplegia type 12 . J Clin Invest. 2012;122(2):538-544.

17. Füger $P$, et al. Spastic paraplegia mutation N256S in the neuronal microtubule motor KIF5A disrupts axonal transport in a Drosophila HSP model. PloS Genet. 2012;8(11):e1003066.

18. Burke RE. Sir Charles Sherrington's the integrative action of the nervous system: a centenary appreciation. Brain. 2007;130(pt 4):887-894.

19. Shepherd GM. Corticostriatal connectivity and its role in disease. Nat Rev Neurosci. 2013;14(4):278-291.

20. Webb S, et al. Autosomal dominant hereditary spastic paraparesis with cognitive loss linked to chromosome 2p. Brain. 1998;121(pt 4):601-609.

\title{
Zinc, insulin, and the liver: a ménage à trois
}

\author{
Thomas V. O'Halloran, ${ }^{1}$ Melkam Kebede, ${ }^{2}$ Steven J. Philips, ${ }^{3}$ and Alan D. Attie ${ }^{2}$ \\ ${ }^{1}$ Chemistry of Life Processes Institute, Departments of Chemistry and Molecular Biosciences, Northwestern University, \\ Evanston, Illinois, USA. ${ }^{2}$ Department of Biochemistry, University of Wisconsin-Madison, Madison, Wisconsin, USA. \\ ${ }^{3}$ Department of Molecular Biosciences, Northwestern University, Evanston, Illinois, USA.
}

\begin{abstract}
Insulin and $\mathrm{Zn}^{2+}$ enjoy a multivalent relationship. $\mathrm{Zn}^{2+}$ binds insulin in pancreatic $\beta$ cells to form crystalline aggregates in dense core vesicles (DCVs), which are released in response to physiological signals such as increased blood glucose. This transition metal is an essential cofactor in insulin-degrading enzyme and several key $\mathrm{Zn}^{2+}$ finger transcription factors that are required for $\beta$ cell development and insulin gene expression. Studies are increasingly revealing that fluctuations in $\mathrm{Zn}^{2+}$ concentration can mediate signaling events, including dynamic roles that extend beyond that of a static structural or catalytic cofactor. In this issue of the JCI, Tamaki et al. propose an additional function for $\mathrm{Zn}^{2+}$ in relation to insulin: regulation of insulin clearance from the bloodstream.
\end{abstract}

Zinc is an abundant and essential element that plays a number of regulatory roles in

Conflict of interest: Thomas V. O'Halloran holds equity positions in Viamet Pharmaceuticals Holdings Inc. and Tactic Pharma. Alan D. Attie was previously a member of the CVMED advisory board for Pfizer

Citation for this article: $J$ Clin Invest. 2013 ; 123(10):4136-4139. doi:10.1172/JCI72325. biology. Studies in model organisms indicate that zinc receptor proteins control complex networks of genes in $\mathrm{Zn}^{2+}$-responsive manners. Moreover, complex developmental events are controlled by dynamic fluctuations of billions of zinc ions between intracellular compartments and extracellular sites $(1,2)$. Secretory compartments enriched in $\mathrm{Zn}^{2+}$ are found in a number of cell types, including hippocampal and olfactory neurons, oocytes, and pancreatic $\beta$ cells. Specific stimulation of these cells leads to $\mathrm{Zn}^{2+}$ exocytosis; however, neither the physiological nor the biochemical roles of the released $\mathrm{Zn}^{2+}$ are yet clear in these systems. In this issue of the JCI, Tamaki et al. describe striking connections among zinc compartmentalization, exocytosis, and insulin uptake by the liver (3).

\section{A link between zinc transport and diabetes}

The ZnT8 transporter (encoded by solute carrier family 30 member 8 gene; SLC30A8) is located on dense core vesicles (DCVs) in $\beta$ cells and loads $\mathrm{Zn}^{2+}$ into these secretory compartments, where it binds with and stabilizes a hexameric form of insulin (4). $\mathrm{ZnT}$ is an autoantigen in type 1 diabetes 